phenotype with worse prognosis. Supported by projects AZV 17-30965A and RVO VFN 61514.

\section{GP225 BETTER OUTCOMES OF 'HIGH-RISK' SCREENED GLUTARIC ACIDURIA TYPE 1 PATIENTS GIVE OPTIMISM TO FUTURE CASES DIAGNOSED THROUGH NEWBORN SCREENING IN IRELAND}

Lydia Healy, Graham King*, Margeurite Lawlor, Joanne Hughes, Ina Knerr, Eileen Treacy, Ellen Crushell, Ahmad Monavari. Temple Street Children's University Hospital, Dublin, Ireland

\subsection{6/archdischild-2019-epa.284}

Introduction Glutaric aciduria Type 1 (GA1), first described in 1975, has an incidence of approximately 1:56,000 in Ireland. GA1 usually presents clinically during an intercurrent illness at 3-18 months' age with encephalopathy and dystonia, resulting in dyskinetic/spastic cerebral palsy and sometimes intellectual disability.

Management of GA1 involves a low-protein diet with synthetic lysine-free/low-tryptophan protein supplement and L-carnitine treatment, with aggressive management of inter-current illnesses to prevent catabolic crisis and encephalopathy. This review assessed outcomes of high-risk (familial) screened Irish patients versus non-screened patients who presented clinically, prior to the very recent introduction of newborn screening (NBS) for GA1 in Ireland.

Method Retrospective chart review of GA1 patients attending the National Centre for Inherited Metabolic Disorders (1981 - 2018). Data was collected to determine patient clinical and neurological status at diagnosis and at school age.

Results 29 patients were diagnosed with and treated for GA1 during the review period (352 patient treatment years). Thirteen $(45 \%)$ presented clinically and $16(55 \%)$ were diagnosed early by 'high-risk screen' due to family history. Median age at diagnosis was 365 days (IQR 206-626) for those presenting clinically and 4.5 days (IQR 3-29) for those screened $(\mathrm{p}<0.001$ Mann-Whitney). Six of twelve (50\%) patients who presented clinically (one was lost to follow-up) are deceased compared to one patient $(6.3 \%)$ in the screened group $(\mathrm{p}=0.008$, Chi-squared).

Eighteen (62\%) patients were from the Irish Travelling Community of which $12(66.7 \%)$ were diagnosed by high-risk screen compared to four of $11(36.4 \%)$ from the non-Traveller population $(\mathrm{P}=0.111$, Chi-squared).

Nine of 15 patients (60\%) diagnosed by high-risk screen had either radiological only or no features of GA1 at diagnosis, compared to one patient $(7.7 \%)$ in the clinically presenting group $(\mathrm{p}=0.004$, Chi-squared).

Two of the eight patients (25\%) presenting clinically who survived to school-age attended mainstream school without significant impairment versus 10 of 14 (71.4\%) screened patients $(\mathrm{p}=0.035$ Chi-squared). Three of eight $(37.5 \%)$ clinical presenters at 5-7 years had either full motor function or a Gross Motor Function Classification System score of 1, compared with all fourteen diagnosed by high-risk screen $(p=0.001$, Chi-squared).

Conclusion Our data supports the necessity to diagnose infants with GA1 in the newborn period and we expect that future outcomes in the Irish NBS population will be similar to this historical high-risk screening group.

\section{GP226 TETRAHYDROBIOPTERIN DISORDERS IN IRELAND: A CASE SERIES OF 7 PATIENTS}

${ }^{1}$ Arie Fisher*, ${ }^{1}$ Ritma Boruah, ${ }^{2}$ Philip D Mayne, ${ }^{1}$ Ahmad A Monavari, ${ }^{1}$ Ellen Crushell, ${ }^{1}$ Ina Knerr. 'National Centre for Inherited Metabolic Disorders, Temple Street Children's University Hospital, Dublin, Ireland; '2Department of Biochemistry, Temple Street Children's University Hospital, Dublin, Ireland

\subsection{6/archdischild-2019-epa.285}

Background Classical Phenylketonuria (PKU) is an autosomal recessively inherited disorder in phenylalanine metabolism caused by profound deficiency of the enzyme phenylalanine hydroxylase (PAH). Newborn screening for PKU was introduced in Ireland in 1966. A low phenylalanine diet is the well-established standard treatment for patients with Classical PKU. However, approximately 1\% of all cases in Ireland are caused by an inborn metabolic defect of the cofactor of $\mathrm{PAH}$, tetrahydrobiopterin (BH4). BH4 is an essential cofactor for various metabolic pathways, including phenylalanine, Dopa and 5-OH-Tryptophan metabolism. This subgroup of ultra-rare neurometabolic diseases is referred to as 'Malignant/Atypical Phenylketonuria', as most of these patients have raised phenylalanine blood levels and develop profound neurological symptoms despite adequate dietary restriction of phenylalanine, if not treated with additional medications to prevent neurotransmitter deficiencies.

Methods We describe diagnostic approaches, biochemical findings and mutational spectrum, clinical presentation, treatment and outcomes in a case series of 7 patients diagnosed with either 6-pyruvoyl-tetrahydrobiopterin synthase (PTPS) deficiency $(n=2)$, a defect in BH4 biosynthesis, or dihydropteridine reductase (DHPR) deficiency $(n=5)$, a defect in $\mathrm{BH} 4$ regeneration.

Results Seven patients aged 2 to 33 years were included in this study after ethics approval was obtained. Six patients were diagnosed in the newborn period; all five patients with DHPR deficiency were diagnosed following detection of an elevated phenylalanine on newborn screening. Four patients had developmental delay and one patient with DHPR deficiency had epilepsy. All five patients with DHPR deficiency were homozygous for c.353C $>\mathrm{T}$ mutation in the QDPR gene. One patient with PTPS deficiency was diagnosed at age 3 years and 9 months when he presented with developmental delay and oculogyric crises, his sibling was subsequently diagnosed as a newborn and is neurologically normal at age 2 years, neither required dietary therapy as their hyperphenylalaninaemia is mild. Both patients with PTPS deficiency are homozygous for c.84-3C $>\mathrm{G}$ mutation in the PTS gene. All patients have been treated with neurotransmitter replacement.

Conclusion Some disorders of $\mathrm{BH} 4$ metabolism such as DHPR deficiency may mimic Classical Phenylketonuria biochemically with a raised blood phenylalanine level, in others, such as PTPS deficiency, the hyperphenylananinaemia may be mild. However, early differential diagnosis is crucial to commence appropriate treatment in a timely manner to improve outcomes in patients with a rare 'Atypical PKU'. These cases demonstrate the importance of $\mathrm{BH} 4$ measurement in patients with a positive PKU newborn screening result and also when evaluating children with unexplained neurological deterioration such as oculogyric crises. 